\title{
Laryngeal mask airway versus endotracheal tube for airway management during laparoscopic percutaneous extraperitoneal closure for inguinal hernia in pediatric day surgery: a retrospective analysis
}

Tamaki Iwade ( $\nabla$ tamakiiwade0203@ceres.ocn.ne.jp)

Osaka Hospital: Osaka Byoin https://orcid.org/0000-0002-1003-8155

Koichi Ohno

Osaka Red Cross Hospital: Osaka Sekijuji Byoin

Research article

Keywords: Day surgery, endotracheal tube, laparoscopic percutaneous extraperitoneal closure, laryngeal mask airway, pediatric laparoscopic surgery

Posted Date: July 19th, 2021

DOI: https://doi.org/10.21203/rs.3.rs-728216/v1

License: (1) This work is licensed under a Creative Commons Attribution 4.0 International License. Read Full License 


\section{Abstract}

\section{Background}

Although endotracheal tube is preferred for airway management during laparoscopic percutaneous extraperitoneal closure for inguinal hernias, laryngeal mask airway may also be used. However, few studies have reported the usefulness of laryngeal mask airway during laparoscopic percutaneous extraperitoneal closure. Our study aims to report the advantages of laryngeal mask airway versus endotracheal tube during laparoscopic percutaneous extraperitoneal closure for inguinal hernia in pediatric day surgery.

Methods

The records of 56 patients (Group I, endotracheal tube; Group II, laryngeal mask airway) treated for inguinal hernia using laparoscopic percutaneous extraperitoneal closure between November 2018 and December 2019 were retrospectively reviewed. The duration of anesthesia; changes in hemodynamics (heart rate and systolic/diastolic blood pressure), end-tidal carbon dioxide, and bispectral index; and postoperative complications were analyzed.

Results

Groups I and II had 39 and 17 patients, respectively. The duration of anesthesia and surgery and changes in hemodynamics and bispectral index were similar between the two groups. Induction and recovery times were significantly shorter and changes in end-tidal carbon dioxide were more significant in Group II $(p<0.05)$. The incidence of sore throat and nausea was higher in Group I $(p<0.05)$.

\section{Conclusions}

Laryngeal mask airway was equivalent to endotracheal tube in terms of performance during laparoscopic percutaneous extraperitoneal closure, although induction and recovery were achieved sooner in laryngeal mask airway, with a lower incidence of sore throat and nausea.

Trial Registration

Not applicable

\section{Background}

An inguinal hernia is one of the most common pediatric surgical problems [1]. Although open repair is a definitive surgery for inguinal hernia, laparoscopic repair has recently been introduced as a less invasive technique. Since its introduction, laparoscopic percutaneous extraperitoneal closure (LPEC) has gained popularity among pediatric surgeons for repairing the inguinal hernia, especially in Japan [2]. Appropriate airway management is important during laparoscopic surgery. However, there is still controversy 
surrounding the use of laryngeal mask airway (LMA) versus an endotracheal tube (ETT), both of which are commonly employed in general pediatric surgery [3]. ETT is considered safer than LMA under general anesthesia, although, in comparison, the advantages of LMA include faster insertion without the use of a laryngoscope and a higher rate of successful first attempts, even for a novice anesthesiologist. Additionally, LMA has been reported to be safe for use in all age groups for various surgical procedures. Thus, LMA has been accepted as a valuable airway device for pediatric anesthesia. However, in laparoscopic surgery, ETT is considered more appropriate than LMA owing to greater difficulty in providing effective ventilation and the risk of gastric inflation involved in LMA. However, a study [4] suggests that LMA provides effective ventilation without the risk of gastric inflation. Furthermore, some studies [3,5-8] suggest that LMA results in an equal or lower incidence of complications, such as cough, desaturation, laryngospasm, sore throat, aspiration, nausea, and vomiting, than ETT. Less invasive laparoscopic techniques for inguinal hernia repair in the pediatric population have recently been introduced; however, few studies have reported the usefulness of LMA during LPEC. Thus, this study aims to compare ETT with LMA during LPEC in pediatric day surgery and to report the safety and effectiveness of LMA.

\section{Methods}

\section{Patients and setting}

A retrospective comparative study was performed at a single institution. All patients who underwent LPEC in the Department of Pediatric Surgery at Hyogo Prefectural Kobe Children's Hospital (Kobe, Japan) between November 2018 and December 2019 were enrolled in the study. Patients meeting the following inclusion criteria were included: (1) those with a history of inguinal hernia treated via LPEC in day surgery, and (2) those with an American Society of Anesthesiologists physical status (ASA-PS) classification of 1 or 2. The exclusion criteria were as follows: (1) no previous abdominal surgery and comorbidities; (2) those with an ASA-PS classification of 3 or higher; and (3) unrecorded changes in hemodynamics (heart rate $[\mathrm{HR}]$ and systolic/diastolic blood pressure [S/DBP]), end-tidal carbon dioxide $\left(\mathrm{ETCO}_{2}\right)$, and bispectral index (BIS) in anesthetic records. The study design was approved by the Institutional Review Board of Hyogo Prefectural Kobe Children's Hospital (approval number: R31-32, on Jan 23, 2020). All parents provided informed consent for the participation of their children in this study.

\section{Study protocol}

All procedures performed were day surgeries; patients were discharged same day. Medical history and demographic data (sex, age, height, weight, and hernia lateralization) were recorded according to the study protocol. Patients were divided into two groups: Group I $(n=39)$, which employed ETT during the procedure, and Group II $(n=17)$, which employed LMA. Anesthesia was performed following the same procedure in both groups. The duration of anesthesia (induction, surgery, and recovery); changes in hemodynamics (HR and S/DBP), ETCO 2 , and BIS; and postoperative complications (laryngospasm, aspiration, sore throat, nausea, and vomiting) were recorded and compared between the two groups. The 
duration of anesthesia (induction, surgery, and recovery) was evaluated as the barometer of surgical performance. Hemodynamics and BIS were evaluated as the barometer of anesthesia management. $\mathrm{ETCO}_{2}$ was evaluated as the barometer of airway management. Induction was defined as the time from oxygen inhalation after entering the operating room to skin incision. Surgery was defined as the time from skin incision to skin suture. Recovery was defined as the time from skin suture to exiting the operating room.

\section{Anesthesiology and surgical technique}

Patients did not receive premedication before entering the operating room, and they entered the operating room with their father or mother. General anesthesia was slowly administered via inhalation using sevoflurane, nitrous oxide, and oxygen. After achieving sufficient depth of anesthesia, an intravenous line was established. Remifentanil hydrochloride, propofol, and rocuronium bromide were administered via an intravenous line. For the LMA group, adequate mask size was important; thus, the largest possible size was placed over the oral cavity. The anesthesiologist inserted LMA (ProSeal; Senko Medical Instrument Mfg. Co., Ltd., Tokyo, Japan) by guiding the tube along the palatopharyngeal curve until resistance was felt, and the cuff was inflated to the appropriate pressure (approximately $20 \mathrm{~cm} \mathrm{H}_{2} \mathrm{O}$ ), allowing ventilation. For the ETT group, the size of the ETT (Shiley; Covidien, CO, USA) for children < 10 years of age was determined by the formula: size $=$ age $/ 4+4$. The tube position was checked using auscultation of both the chest and abdomen to ensure that both the lungs were equally ventilated. The ETT was secured with two pieces of adhesive tape placed around the oral cavity and the tube. After establishing the airway, an ultrasound-guided transversus abdominis plane block was used on all patients. Before extubation or the removal of LMA, sugammadex sodium was administered via an intravenous line to recover from muscle relaxation. All operations were performed with patients in the supine position using LPEC for laparoscopic inguinal hernia repair as described previously [2]. Insufflation pressure was set between 8-10 $\mathrm{cm} \mathrm{H} \mathrm{H}_{2} \mathrm{O}$ for all patients.

\section{Statistical analyses}

Relevant data were obtained by reviewing patients' medical records and were analyzed using SPSS 26.0 software (IBM Corp., Armonk, NY, USA). The mean \pm standard deviation was used for descriptive statistics. The data comprising continuous variables between the groups of patients were analyzed using the Student's $t$-test, and categorical variables were analyzed using the Chi-square test. A $p$-value $<0.05$ was considered statistically significant.

\section{Results}

Overall, 64 patients were included, of which eight did not meet the inclusion criteria because of unrecorded changes in hemodynamics (HR and S/DBP), $\mathrm{ETCO}_{2}$, or BIS in the anesthetic records. Finally, 56 patients were eligible for assessment. Group I (ETT) had 39 patients (70\%), while Group II (LMA) had 17 patients (30\%). Patients' demographics and duration of anesthesia are summarized in Table 1. No significant differences between the two groups were observed for age $(p=0.244)$, height $(p=0.321)$, or 
body weight $(p=0.549)$. No significant differences between the two groups were observed during anesthesia and surgery. Induction and recovery times were significantly shorter in Group II $(28.3 \pm 4.86 \mathrm{vs}$. $25.2 \pm 3.44$ mins, $p=0.030 ; 12.9 \pm 5.49$ vs. $8.9 \pm 4.40$ mins, $p=0.011$ ).

Table 1

Patients' demographics and duration of anteshesia

\begin{tabular}{|c|c|c|c|}
\hline & 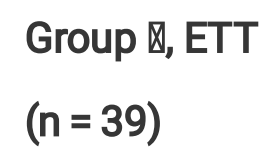 & $\begin{array}{l}\text { Group } \bigotimes, \text { LMA } \\
(n=17)\end{array}$ & $p$-value \\
\hline \multicolumn{4}{|l|}{ Patients' demographics } \\
\hline Sex (male/female) & $18 / 21$ & $2 / 15$ & - \\
\hline Hernia lateralization (R/L/Bil) & $13 / 7 / 19$ & $7 / 3 / 7$ & - \\
\hline Age (months) & $65.0 \pm 34.97$ & $75.5 \pm 27.74$ & 0.244 \\
\hline Height (cm) & $107.9 \pm 19.87$ & $112.7 \pm 13.76$ & 0.321 \\
\hline Body weight (kg) & $19.2 \pm 10.22$ & $20.4 \pm 5.84$ & 0.549 \\
\hline \multicolumn{4}{|l|}{ Duration (minutes) } \\
\hline Anesthesia & $71.7 \pm 10.59$ & $65.8 \pm 9.19$ & 0.062 \\
\hline Induction & $28.3 \pm 4.86$ & $25.2 \pm 3.44$ & $0.030^{*}$ \\
\hline Surgery & $30.5 \pm 7.14$ & $31.5 \pm 9.36$ & 0.582 \\
\hline Recovery & $12.9 \pm 5.39$ & $8.9 \pm 4.40$ & $0.011^{*}$ \\
\hline \multicolumn{4}{|c|}{$\begin{array}{l}\text { a Student's } t \text {-test. }{ }^{*} p<0.05 \text { (indicates statistically significant differences between the groups). Data } \\
\text { are presented as the mean } \pm \text { standard deviation. }\end{array}$} \\
\hline
\end{tabular}

The hemodynamics (HR and S/DBP) during the procedure are summarized in Table 2. No significant differences between the two groups were observed in HR and DBP. The maximum SBP was significantly higher in Group II ( $96.5 \pm 8.55$ vs. $101.9 \pm 9.20 \mathrm{mmHg}, p=0.048)$. Additionally, for changes in hemodynamics, no significant differences were observed between the two groups. The maximum BIS value was significantly higher in Group II than in Group I ( $65.5 \pm 5.32$ vs. $69.1 \pm 4.35 ; p=0.023)$; however, there was no significant between-group difference in the minimum BIS and change in BIS. 
Table 2

Hemodynamics and BIS during LPEC

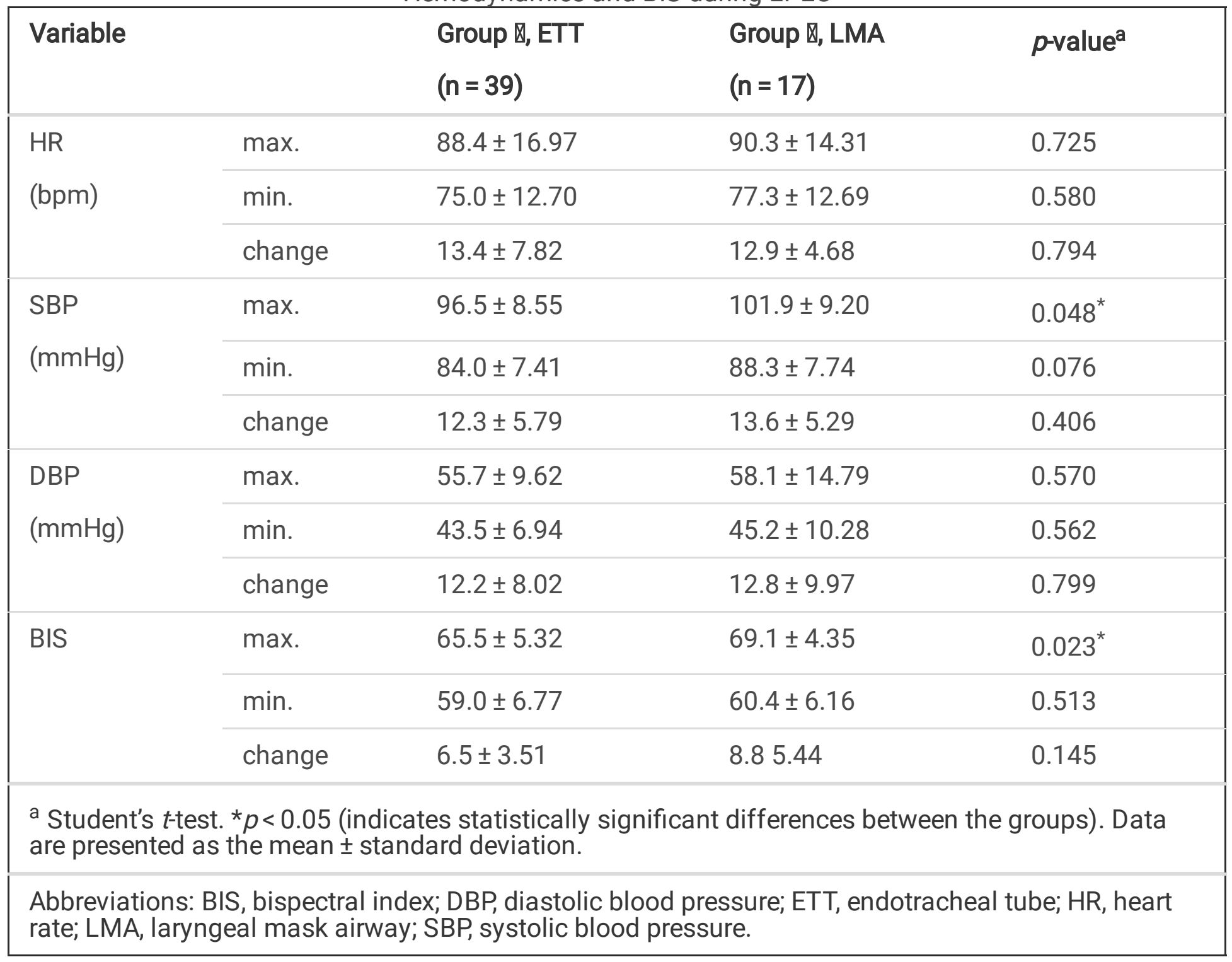

$\mathrm{ETCO}_{2}$ and $\mathrm{BIS}$ during the procedure are summarized in Table 3 . There were no differences in the minimum $\mathrm{ETCO}_{2}$ value between the two groups; however, the maximum $\mathrm{ETCO}_{2}$ value and change in $\mathrm{ETCO}_{2}$ were more significant in Group II than in Group I $(43.8 \pm 3.10$ vs. $46.9 \pm 5.24 \mathrm{mmHg} ; p=0.036 ; 7.1$ \pm 2.99 vs. $10.4 \pm 4.23 \mathrm{mmHg} ; p=0.008$ ). 
Table 3

$\mathrm{ETCO}_{2}$ during LPEC

\begin{tabular}{|c|c|c|c|c|}
\hline Variable & & $\begin{array}{l}\text { Group } \otimes, \text { ETT } \\
(n=39)\end{array}$ & $\begin{array}{l}\text { Group } \mathrm{X}, \text { LMA } \\
(\mathrm{n}=17)\end{array}$ & $p$-value ${ }^{a}$ \\
\hline \multirow[t]{3}{*}{$\mathrm{ETCO}_{2}(\mathrm{mmHg})$} & $\max$. & $43.8 \pm 3.10$ & $46.9 \pm 5.24$ & $0.036^{*}$ \\
\hline & $\min$. & $36.7 \pm 3.28$ & $36.4 \pm 2.61$ & 0.775 \\
\hline & change & $7.1 \pm 2.99$ & $10.4 \pm 4.23$ & $0.008^{*}$ \\
\hline \multirow[t]{3}{*}{ BIS } & $\max$. & $65.5 \pm 5.32$ & $69.1 \pm 4.35$ & $0.023^{*}$ \\
\hline & $\min$. & $59.0 \pm 6.77$ & $60.4 \pm 6.16$ & 0.513 \\
\hline & change & $6.5 \pm 3.51$ & 8.85 .44 & 0.145 \\
\hline
\end{tabular}

a Student's $t$-test. ${ }^{*} p<0.05$ (indicates statistically significant differences between the groups). Data are presented as the mean \pm standard deviation.

Abbreviations: $\mathrm{ETCO}_{2}$, end-tidal carbon dioxide; ETT, endotracheal tube; LMA, laryngeal mask airway; LPEC, laparoscopic percutaneous extraperitoneal closure.

The postoperative complications are summarized in Table 4. There were significant differences in nausea and sore throat in Group I ( $p=0.020$ and 0.018 , respectively), but no significant differences in laryngospasm, aspiration, and vomiting.

Table 4

Postoperative complications

\begin{tabular}{|c|c|c|c|}
\hline Complication & $\begin{array}{l}\text { Group } \rrbracket, E T T \\
(n=39)\end{array}$ & $\begin{array}{l}\text { Group } \otimes, \text { LMA } \\
(n=17)\end{array}$ & $p$-value ${ }^{a}$ \\
\hline Laryngospasm & $0(0.0)$ & $0(0.0)$ & - \\
\hline Aspiration & $0(0.0)$ & $0(0.0)$ & - \\
\hline Sore throat & $5(12.8)$ & $0(0.0)$ & $0.018^{*}$ \\
\hline Nausea & 7 (17.9) & $1(5.9)$ & $0.020^{*}$ \\
\hline Vomiting & $2(5.1)$ & $0(0.0)$ & 0.148 \\
\hline
\end{tabular}

\section{Discussion}


The main findings of this study were that safe management of anesthesia and shorter induction and recovery times with lower postoperative complications could be achieved with LMA.

A recent study [4] reported the safety of LMA in laparoscopic surgery among both adults and children. Our study indicated that induction and recovery times were significantly shorter in the LMA group; however, there were no significant differences in the duration of anesthesia or surgery between the two groups. Nevešćanin et al. [5] reported that LMA has the advantage of securing patients' airways faster than ETT because a laryngoscope is not needed, and there is a higher rate of successful first attempts; these advantages suggested that induction was significantly shorter using LMA in our study. Our study indicated that LMA using a muscle relaxant proceeded to decrease recovery times compared to ETT. Although Ahiskalioglu et al. [6] reported that recovery times were significantly shorter for LMA without using a muscle relaxant, Tulgar et al. [4] reported that there was a significant difference between ETT and LMA using a muscle relaxant during recovery. Using a muscle relaxant was beneficial in terms of the clinical performance of LMA in pediatric patients under general anesthesia [4, 7]. Our study suggested that LMA may provide and maintain the same visibility as ETT to secure surgical safety and may shorten induction and recovery times using a muscle relaxant, in agreement with previous reports $[4,7]$. Despite these results, ETT continues to be preferred over LMA owing to easier airway management and low risk of abdominal insufflation $[4,5]$.

Changes in hemodynamics were not significantly different between the LMA and ETT groups in our study. In previously reported adult laparoscopic surgeries, no significant changes in hemodynamic responses (HR and mean BP) between the LMA and ETT groups were observed [8]. Previous studies [3, 9] on general pediatric surgery reported that the hemodynamic responses were lower when using LMA. However, in pediatric laparoscopic surgery, our study was the first to compare hemodynamic responses between the LMA and ETT groups. In our study, although the HR and S/DBP were elevated in the LMA group, no significant differences in the changes in HR and S/DBP were observed between the LMA and ETT groups. This suggests that, although the elevation in HR and S/DBP in the LMA group was observed during LPEC, the changes in HR and S/DBP may be stable with either device. Thus, we suggest that elevation of hemodynamics with LMA could be permissive during LPEC.

BIS is a processed electroencephalographic parameter that quantifies the depth of anesthesia. It is represented by a number between 0 and 100, with 0 representing deep anesthesia and 100 indicating complete consciousness; the depth of general anesthesia and the depth of deep sedation are represented by BIS values of $40-60$ and $60-70$, respectively. Although BIS in pediatric anesthesia may be used to guide anesthetic administration in children $>2$ years of age, BIS is not recommended for infants $<6$ months of age $[10,11]$. The age of the patients in the ETT and LMA groups were $65.0 \pm 34.97$ and $75.5 \pm$ 27.74 months, respectively. Therefore, we believe that BIS was reliable for measuring the depth of anesthesia. The BIS values in our study ranged between $59.0 \pm 6.77$ and $65.5 \pm 5.32$ in the ETT group and between $60.4 \pm 6.16$ and $69.1 \pm 4.35$ in the LMA group. These results showed that the depth of anesthesia corresponded with deep sedation in the two groups. The change in BIS was $6.5 \pm 3.51$ and $8.8 \pm 5.44$ in the ETT and LMA groups, respectively; no significant difference was observed. These findings suggested 
that the same depth of anesthesia may be achieved with either device. Sinha et al. [11] reported that the removal of LMA during deep sedation (median BIS, 60; range, 58-76) reduced the prevalence of airway complications. Our finding that intraoperative BIS values between $60.4 \pm 6.16$ and $69.1 \pm 4.35$ corresponded with deep sedation may be a suitable indicator for the removal of LMA during LPEC owing to a low incidence of postoperative complications with LMA. Furthermore, our study suggests that during LPEC with LMA, the depth of anesthesia may be managed by deep sedation.

The maximum $\mathrm{ETCO}_{2}$ value and change in $\mathrm{ETCO}_{2}$ were significantly higher between the LMA and ETT groups in our study. Previous studies $[3,6,8,9]$ of general or laparoscopic surgery in adult or pediatric patients reported that no significant difference in the change in $\mathrm{ETCO}_{2}$ was observed between the two devices. Our findings were not consistent with the findings reported in previous studies. However, the maximum $\mathrm{ETCO}_{2}$ value in the LMA group was close to the upper normal limit of $\mathrm{ETCO}_{2}$. Thus, we supposed that the elevation in $\mathrm{ETCO}_{2}$ did not affect surgical performance and airway management, if the maximum $\mathrm{ETCO}_{2}$ value was close to the upper normal limit of $\mathrm{ETCO}_{2}$.

In our study, there was a greater incidence of postoperative complications, such as nausea and sore throat, with ETT. Additionally, previous studies $[3,8,12]$ reported that ETT was associated with a greater incidence of sore throat (13.3-54.7\%) than LMA; our finding was consistent with those of previous studies. In our study, we did not observe laryngospasm and aspiration in all cases; however, such complications were reported in previous studies $[5,8,12]$.

Our study has two limitations. First, it was a retrospective comparative study. Second, the study was conducted in a single institution with a small study sample. However, the aim was to compare the duration of anesthesia and the changes in vital signs, $\mathrm{ETCO}_{2}$, and $\mathrm{BIS}$, and postoperative complications. Additionally, the sample size was determined based on previous studies. Future studies with larger sample sizes are required to accurately determine the usefulness of LMA during LPEC in pediatric day surgery. While no previous reports compare changes in BIS achieved while using ETT versus LMA during LPEC, our study revealed that LMA was able to maintain the same quality and depth of anesthesia as ETT. Furthermore, while the use of LMA was equivalent to that of ETT with respect to the changes in hemodynamics and BIS, induction and recovery times were shorter when using LMA, with a low incidence of postoperative complications.

\section{Conclusions}

LMA may be considered safe and useful during LPEC in pediatric day surgery. The airway management of LMA was considered be an aid for minimally invasive laparoscopic day surgery in children.

\section{Abbreviations}

ASA-PS, American Society of Anesthesiologists physical status; BIS, bispectral carbon dioxide; CONSORT, Consolidating Standards of Reporting Trials; DBP, diastolic blood pressure; ETCO ${ }_{2}$, end-tidal carbon 
dioxide; ETT, endotracheal tube; HR, heart rate; LMA, laryngeal mask airway; LPEC, laparoscopic percutaneous extraperitoneal closure; SBP, systolic blood pressure.

\section{Declarations}

Ethics approval and consent to participate: The study was approved by the Institutional Review Board of Hyogo Prefectural Kobe Children's Hospital (approval number: R31-32. Jan 23, 2020). All parents provided informed consent for the participation of their children in this study.

Consent for publication: Not applicable.

Availability of data and materials: The datasets used and/or analyzed during the current study are available from the corresponding author on reasonable request.

Competing interests: The authors declare that they have no competing interests.

Funding: This research did not receive any specific grant from funding agencies in the public, commercial, or not-for-profit sectors.

Authors' contributions: TI designed the study, collected and analyzed data, and wrote the manuscript; KO reviewed the manuscript and supervised the study. All authors read and approved the final manuscript.

Acknowledgments: We would like to thank Editage (www.editage.com) for their writing support.

\section{References}

1. Ein SH, Njere I, Ein A. Six thousand three hundred sixty-one pediatric inguinal hernias: a 35-year review. J Pediatr Surg. 2006;41:980-6.

2. Miyake H, Fukumoto K, Yamoto M, Nouso H, Kaneshiro M, Nakajima H, et al. Comparison of percutaneous extraperitoneal closure (LPEC) and open repair for pediatric inguinal hernia: experience of a single institution with over 1000 cases. Surg Endosc. 2016;30:1466-72.

3. Patel MG, Swadia V, Bansal G. Prospective randomized comparative study of use of PLMA and ET tube for airway management in children under general anaesthesia. Indian J Anaesth. 2010;54:10915.

4. Tulgar S, Boga I, Cakiroglu B, Thomas DT. Short-lasting pediatric laparoscopic surgery: Are muscle relaxants necessary? Endotracheal intubation vs. laryngeal mask airway. J Pediatr Surg. 2017;52:1705-10.

5. Nevešćanin A, Vickov J, Elezović Baloević S, Pogorelić Z. Laryngeal mask airway versus tracheal intubation for laparoscopic hernia repair in children: Analysis of respiratory complications. $J$ Laparoendosc Adv Surg Tech A. 2020;30:76-80.

6. Ahiskalioglu A, İnce İ, Ahiskalioglu EO, Oral A, Aksoy M, Yiğiter M, et al. Is neuromuscular blocker necessary in pediatric patients undergoing laparoscopic inguinal hernia repair with percutaneous 
internal ring suturing? Eur J Pediatr Surg. 2017;27:263-8.

7. Byun SH, Kim SJ, Kim E. Comparison of the clinical performance of the flexible laryngeal mask airway in pediatric patients under general anesthesia with or without a muscle relaxant: study protocol for a randomized controlled trial. Trials. 2019;20:31.

8. Parikh SS, Parekh SB, Doshi C, Vyas V. ProSeal laryngeal mask airway versus cuffed endotracheal tube for laparoscopic surgical procedures under general anesthesia: A random comparative study. Anesth Essays Res. 2017;11:958-63.

9. Sinha A, Sharma B, Sood J. ProSeal as an alternative to endotracheal intubation in pediatric laparoscopy. Pediatr Anesth. 2007;17:327-32.

10. Ganesh A, Watcha MF. Bispectral index monitoring in pediatric anesthesia. Curr Opin Anaesthesiol. 2004;17:229-34.

11. Sinha A, Sood J. Safe removal of LMA in children - at what BIS? Pediatr Anesth. 2006;16:1144-7.

12. Miskovic A, Johnson M, Frost L, Fernandez E, Pistorio A, Disma N. A prospective observational cohort study on the incidence of postoperative sore throat in the pediatric population. Pediatr Anesth. 2019;29:1179-85. 